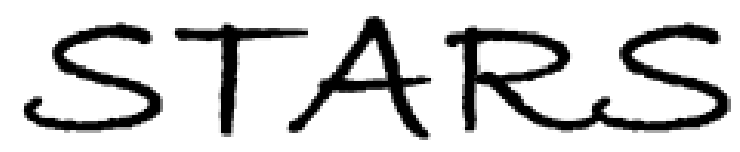

University of Central Florida

STARS

$1-1-1993$

\title{
Relative Chirality Of Octupolar Columns In A Triangular Array
}

\author{
M. L. Plumer
}

A. Caillé

O. Heinonen

University of Central Florida

Find similar works at: https://stars.library.ucf.edu/facultybib1990

University of Central Florida Libraries http://library.ucf.edu

This Article is brought to you for free and open access by the Faculty Bibliography at STARS. It has been accepted for inclusion in Faculty Bibliography 1990s by an authorized administrator of STARS. For more information, please contact STARS@ucf.edu.

\section{Recommended Citation}

Plumer, M. L.; Caillé, A.; and Heinonen, O., "Relative Chirality Of Octupolar Columns In A Triangular Array" (1993). Faculty Bibliography 1990s. 876.

https://stars.library.ucf.edu/facultybib1990/876

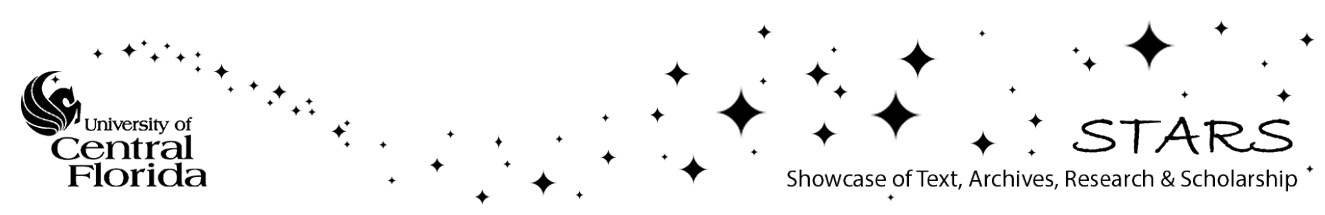




\title{
Relative chirality of octupolar columns in a triangular array
}

\author{
M. L. Plumer and A. Caillé \\ Centre de Recherche en Physique du Solide et Département de Physique, Université de Sherbrooke, \\ Sherbrooke, Québec, Canada J1K $2 R 1$ \\ O. Heinonen \\ Department of Physics, University of Central Florida, Orlando, Florida 32816
}

(Received 10 August 1992)

\begin{abstract}
The relative chirality of helical columns of planar disks having threefold symmetry is studied using a model Hamiltonian derived from symmetry arguments that describe low-order octupole interactions. The columns are assumed to pack in a triangular array perpendicular to the columnar axes. Groundstate and finite-temperature mean-field phase diagrams are obtained as functions of interaction parameters. Due to the structure of the disks, there appears a term in the Hamiltonian that is not present for systems with lower internal symmetry, such as classical spin systems. This interaction is responsible for the stabilization of phases in which one third of the columns have a chirality opposite to the others. Such a phase has been observed in the discotic liquid crystal hexa-hexylthiotriphenylene.
\end{abstract}

\section{INTRODUCTION}

Studies of effects associated with geometrical frustration and chirality have proven important in attempts to further reveal the nature of ordered states and phase transitions in magnetic ${ }^{1-3}$ and polymer ${ }^{4,5}$ systems with triangular coordination. Such concepts are also useful in the understanding of orientational and positional ordering in the hexa- $n$-alkylthiotriphenylene liquid crystals. ${ }^{6-9}$ The disklike molecules in these materials have stiff planar cores linked by thiol groups to 6-carbon alkyl tails. A1though a number of different phase-transition sequences have been observed in related compounds with different tail lengths, ${ }^{9}$ the present work is concerned with HHTT, hexa-hexylthiortriphenylene, the compound as described in Refs. 6 and 7. As the temperature is lowered from the isotropic phase $\mathrm{I}$, there is an initial transition at $T \sim 93^{\circ} \mathrm{C}$ to the $D_{h d}$ state where the disks become arranged in a triangular configuration but with no long-range positional or orientational order along the columns. At $T \sim 70^{\circ} \mathrm{C}$ there is another transition to the $H$ (also called $D_{h 0}$ ) phase where positional and helical ordering of the disks set in along the columns, and with additional modifications of the structure as discussed below. ${ }^{7}$ Finally, a monoclinic $K$ phase appears at $T \sim 62^{\circ} \mathrm{C}$. (Note that both $H$ and $K$ phases have long-range order in all three dimensions, and although thermal fluctuations remain relatively large, these states are not strictly liquid crystalline. ${ }^{9,10}$ ) This paper describes a model of the $H$ phase (and to a limited extent the $H-D_{h d}$ transition), in particular the relative chirality of the columns.

A unique feature of these compounds is the threefold symmetry of the disks. Thus, in contrast with the magnetic-dipole moment of spin systems ${ }^{2}$ or previously studied quadrupole moments of certain polymers, ${ }^{4}$ the lowest-order moment of the mass distribution of HHTT is octupolar. We demonstrate here that objects with this symmetry occupying sites on a hexagonal lattice form a system which allows for terms in the Hamiltonian (not present in spin models) that are crucial in determining the relative chirality of neighboring columns. Such terms partially account for the frustrating effects of the interdigitation of hydrocarbon tails (cf. interlocking of gears) for disks on a triangular lattice, a feature of these materials emphasized in Refs. 6-8. We believe this to be the first attempt to model these effects. The present work also serves to compliment a previous investigation of the thermodynamics of single columns ${ }^{11}$ and also to provide a basis for more realistic treatments of thermal phase transitions when intercolumn interactions are included.

In the $H$ phase of HHTT, the molecules are stacked in columns which are packed in a triangular configuration. Some of the frustration is relieved ${ }^{6-8}$ by a displacement of one-third of the columns along the $c$ axis by half of the interplanar spacing, $z=\frac{1}{2} c$. The resulting configuration, shown in Fig. 1, consists of bilayers which form interpenetrating honeycomb and triangular lattices. The new unit cell has a basal-plane lattice constant $a=\sqrt{3} a_{0}$, where $a_{0}$ corresponds to the original simple hexagonal lattice. A similar structure of magnetic ions occurs in the quasi-one-dimensional antiferromagnet $\mathrm{RbFeBr}_{3}$; $^{12}$ certain features of the magnetic phase transitions in that material are similar to results described in the present work. Because of the two interpenetrating sublattices, two types of basal-plane interactions are considered here (as in Ref. 12), between nearest neighbors within the honeycomb lattice and those between neighboring honeycomb and triangular sites. It is noteworthy that all five of the possibilities for relative chirality and orientational order in the $H$ phase of HHTT considered in Ref. 7 are a feature of the present model with only these parameters.

This paper is organized as follows. In the next section, a model Hamiltonian is derived from symmetry arguments for a system which contains some relevant charac- 


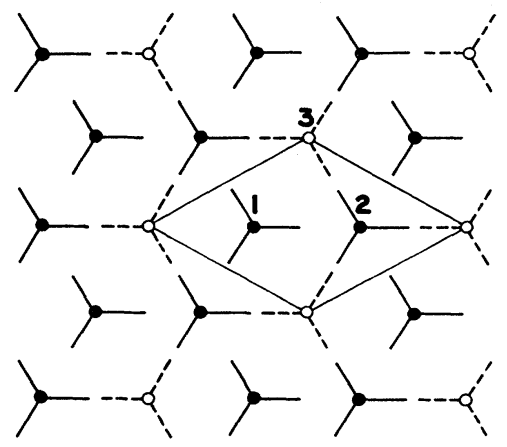

FIG. 1. Schematic of the basal-plane octupole sites where filled circles form a honeycomb lattice at $z=0$ (sites 1 and 2) and open circles (sites 3 ) represent sites displaced by $z=\frac{1}{2} c$, arranged on a triangular lattice. In this particular configuration, octupoles on the displaced sites are rotated by $60^{\circ}$. The basalplane unit cell is indicated by the thin line.

teristics in common with HHTT. Illustrative groundstate phase diagrams featuring different relative chirality states as a function of interaction parameters are given in Sec. III. Analysis of a Landau-type free energy based on a molecular-field approximation provides a simple treatment of thermal effects examined in Sec. IV. Conclusions and a discussion of the results are given in Sec. V.

\section{MODEL HAMILTONIAN}

For the limited purposes of this work, it is assumed that the disklike octupoles (or rotors) are rigid and that they lie perpendicular to the $c$ axis on the rigid lattice sites as shown in Fig. 1. (Further remarks are made regarding this assumption in the following sections.) It is then convenient to construct a suitable Hamiltonian which accounts for orientational degrees of freedom by starting from multipole expansion of the rotor mass distribution, ${ }^{4}$

$$
\begin{aligned}
M\left(\rho, \theta-\theta_{i}, z\right)=\sum_{m=0}[ & A_{m}(\rho, z) \cos m\left(\theta-\theta_{i}\right) \\
& \left.+B_{m}(\rho, z) \sin m\left(\theta-\theta_{i}\right)\right],
\end{aligned}
$$

where $(\rho, \theta, z)$ are cylindrical coordinates and $\theta_{i}$ describes the angle of one arm of the rotor relative to the hexagonal $a$ axis, as shown in Fig. 2. The lowest-order term in this expansion is $m=3$ (octupole moment), due to the threefold symmetry of the disks. Invariance with respect to the $D_{3}$ point-group symmetry of the disks in situ ${ }^{7}$ also requires that $A(\rho,-z)=A(\rho, z)$ and $B(\rho,-z)$ $=-B(\rho, z)$. The Hamiltonian is constructed from octupole moments calculated using the definition

$$
\psi_{l m}=4 /(\pi \rho)^{3} \int_{-\pi}^{\pi} d \theta x^{1} y^{m} M\left(\rho, \theta-\theta_{i}, z\right),
$$

where $l+m=3$ to lowest order. The following relations are a result of this expression:

$$
\psi_{30}=\psi_{x x x}=A \cos 3 \theta_{i}-B \sin 3 \theta_{i},
$$

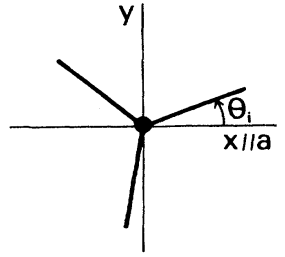

FIG. 2. Schematic showing the angle $\theta_{i}$ which defines the ocutpole rotor orientation relative to the hexagonal $\hat{\mathbf{a}} \| \hat{\mathbf{x}}$ axis.

$$
\psi_{21}=\psi_{x x y}=A \sin 3 \theta_{i}+B \cos 3 \theta_{i},
$$

and $\psi_{12}=-\psi_{30}, \psi_{03}=-\psi_{21}$.

The Hamiltonian is constructed to be invariant with respect to hexagonal symmetry. This requirement yields two independent terms, which may be expressed as

$$
\begin{aligned}
H= & -\frac{1}{2} \sum_{i j} J\left(\mathbf{R}_{i}-\mathbf{R}_{j}\right)\left[\psi_{x x x}\left(\mathbf{R}_{i}\right) \psi_{x x x}\left(\mathbf{R}_{j}\right)\right. \\
& \left.+\psi_{y y y}\left(\mathbf{R}_{i}\right) \psi_{y y y}\left(\mathbf{R}_{j}\right)\right] \\
& -\frac{1}{2} \sum_{i j} G\left(\mathbf{R}_{i}-\mathbf{R}_{j}\right) \psi_{y y y}\left(\mathbf{R}_{i}\right) \psi_{y y y}\left(\mathbf{R}_{j}\right) .
\end{aligned}
$$

In an effort to provide a simple model for relative chirality effects, it is assumed here that the disklike molecules are flat (and not the propeller blade structure discussed in Refs. 6-9) so that $z \rightarrow-z$ symmetry of the mass density is imposed. This requirement gives $B=0$. With relations (3) and (4), the Hamiltonian can then be expressed as

$$
\begin{aligned}
H\left[\theta_{i}\right]= & -\frac{1}{2} \sum_{i j} J\left(\mathbf{R}_{i}-\mathbf{R}_{j}\right) \cos 3\left(\theta_{i}-\theta_{j}\right) \\
& -\frac{1}{2} \sum_{i j} G\left(\mathbf{R}_{i}-\mathbf{R}_{j}\right) \sin 3 \theta_{i} \sin 3 \theta_{j},
\end{aligned}
$$

where we set $A \equiv 1$ and note that the second term could be equivalently written as a product of cosines or as the sum $\cos 3\left(\theta_{i}-\theta_{j}\right)+\cos 3\left(\theta_{i}+\theta_{j}\right)$. Single-site anisotropy, which has the form ${ }^{4,5,11} \gamma \sum_{i} \cos n \theta_{i}$ (where $n=3$ or $n=6$ ), is omitted from the present simple model; investigations of such anisotropy effects in the case of single mean-field coupled columns are continuing. ${ }^{5,11,13}$ It is noteworthy here that the usual signature of significant anisotropy effects in helical structures (a nonconstant interlayer turn angle, which induces higher harmonics in the structure factor) has not been observed in HHTT; however, such harmonics may be difficult to detect due to the relatively large thermal motion of the molecules along the $c$ axis. ${ }^{14}$

The second term in (5) and (6) ( $G$ term) as derived above does not appear in previous studies of related disklike systems. A different development of a model Hamiltonian for the study of quadrupole-moment columns in an unspecified two-dimensional configuration is presented in Ref. 4. In that work, terms are generated by the contraction of indices in the sum over quadrupole products. Such a scheme yields only isotropic terms, and in the 
present case gives only the $J$ term of (5) and (6). Our approach is specific to the underlying symmetry of the lattice and generates all invariants. (In the case of quadrupole moments, a $G$-type term of the form $\sum_{i j} G_{i j} \cos 2 \theta_{i} \cos 2 \theta_{j}$ exists for square lattices). Other interactions considered in Ref. 4 which arise from explicit coupling to the lattice, not included in the present work, do in fact yield a term of the same form as the $G$ term given above for octupoles in a triangular array. Note that with the factor 3 replaced by 1 , the first term in the Hamiltonian (6) is just that encountered in the much studied planar model of (dipolar) spin systems. The effect of the $G$ term in (6) (not present in magnetic Hamiltonians) on the relative chirality of octupolar columns is emphasized in this work.

\section{GROUND-STATE PHASES}

Relative chirality states at zero temperature are considered here using the model Hamiltonian (6) with only close neighbor interactions included. The unit cell contains three sites, labeled $1,2,3$ in Fig. 1. Two types of basal-plane couplings will be accounted for, between sites 1-2: $J_{\perp}$ and $G_{1}$, as well as between sites $1-3$ and equivalently 2-3: $\widetilde{J}_{\perp}^{\prime}$ and $\widetilde{G}_{\perp}^{\prime}$ (also see Ref. 12). Assuming a constant interlayer turn angle $\alpha=\theta_{n}-\theta_{n-1}$ for the helical configuration of disks along the $c$ axis, the basalplane interactions for the three columns can be expressed by

$$
\begin{aligned}
H_{\perp}= & -J_{\perp} \sum_{n} \cos \left(\phi_{1 n}-\phi_{2 n}\right)-G_{\perp} \sum_{n} \sin \phi_{1 n} \sin \phi_{2 n} \\
& -\widetilde{J}_{\perp}^{\prime} \cos \left[\frac{3}{2} \alpha\right] \sum_{n}\left[\cos \left(\phi_{1 n}-\phi_{3 n}\right)+\cos \left(\phi_{2 n}-\phi_{3 n}\right)\right] \\
& -\widetilde{G}_{\perp}^{\prime} \cos \left(\frac{3}{2} \alpha\right) \sum_{n}\left(\sin \phi_{1 n}+\sin \phi_{2 n}\right) \sin \phi_{3 n}
\end{aligned}
$$

where $n$ labels sites along the $c$ axis, and for convenience we define $\phi=3 \theta$. The factors $\cos \left(\frac{3}{2} \alpha\right)$ arise from the coupling of sites 1 and 2 to the two equidistant sites on chain 3 located at $z= \pm \frac{1}{2} c$.

A variety of different types of interactions may be assumed to stabilize the helical orientation of disks along the $c$ axis. In so-called chiral models, an interaction of the form 5,11

$$
H_{\|}=-J_{\|} \sum_{n} \cos \left(\phi_{n}-\phi_{n-1}-3 \alpha\right)
$$

yields a constant interlayer turn angle $\alpha$. The same state may occur in a nonchiral model with ordinary first- and second-neighbor interactions as described by the first term in $(6)^{15}$,

$$
H_{\|}=-J_{1} \sum_{n} \cos \left(\phi_{n}-\phi_{n-1}\right)-J_{2} \sum_{n} \cos \left(\phi_{n}-\phi_{n-2}\right),
$$

with $\alpha$ determined by $\cos 3 \alpha=-J_{1} /\left(4 J_{2}\right)$. The chirality, right or left handed, is determined by the sign of $\alpha$ in the Hamiltonian (8) but is unspecified by the form (9). For crystal structures which contain a center of inversion symmetry, such as HHTT, the Hamiltonian (9) is strictly more appropriate. $\left[\mathrm{CsCuCl}_{3}\right.$ is an example of a helical system exhibiting a preferred handeness described by (8). ${ }^{16}$ ] This difference may be of importance for thermodynamic quantities for which excitations involving dynamic helicity reversal are important. ${ }^{14,15}$ Chiral degeneracy may be artificially introduced into (8) by the replacement $\alpha \rightarrow \pm|\alpha|$ so that either model may be assumed for the purposes of the present work.

The value of interlayer turn angle $\alpha$ determined for HHTT is sensitive to sample purity and experimental conditions. The result reported in Refs. $6-8$ for samples of free-standing strands is $45.5^{\circ}$, incommensurate with the underlying lattice. A more recent study ${ }^{9}$ on samples of HHTT powder revealed an exactly commensurate value of $\alpha=2 \pi / 8$. The difference is attributed to surface-tension effects present in the strands. It is of interest to note that semiempirical conformational analysis $^{17}$ of dimers similar to HHTT yields a relative orientation of about $45^{\circ}$. There is, however, a natural tendency for orientational order to be commensurate for molecules lying on regular lattice sites. ${ }^{18}$ This effect arises from the presence of Umklapp terms of the form $\delta(m \mathbf{Q}-\mathbf{G})$, where $\mathbf{Q}$ denotes the orientational periodicity and $\mathbf{G}$ is a reciprocal-lattice vector, in the Fourier expansion of the intermolecular potential. Higher-order terms, e.g., the $m=8$ term of interest here, are usually small but can be important if $\mathbf{Q}$ is already nearly commensurate, as in the present case. For free-standing strands, surface tension forces apparently dominate over these Umklapp terms. (Analogous effects also appear in magnetic systems; see, e.g., Ref. 19). For the purposes of the present work, it is only important that $3 \alpha$ does not have a value $m \pi$ (see below).

The relative chirality of the three columns is determined by the parameters $J_{\perp}, G_{\perp}, J_{\perp}^{\prime} \equiv \cos \left(\frac{3}{2} \alpha\right) \widetilde{J}_{\perp}^{\prime}, G_{\perp}^{\prime}$ $\equiv \cos \left(\frac{3}{2} \alpha\right) \widetilde{G}_{1}^{\prime}$ (but is not affected by parameters in $H_{\|}$). The orientation of the disk on column $p$ at layer $n$ is assumed to have the form

$$
\theta_{p n}=\theta_{p}+n \alpha c_{p}
$$

where $\theta_{p}$ gives the relative orientation at layer 0 and $c_{p}= \pm 1$ indicates the chirality. (The definition $\phi_{\mathrm{pn}} \equiv 3 \theta_{\mathrm{pn}}$ is used below.) There are only three independent configurations of relative chirality for the three columns, ,+++++- , and -++ , which indicate the values of $\left\{c_{1} c_{2} c_{3}\right\}$. After performing the sums over $n$ in expression (7), the ground-state energies per disk for these three states are given by

$$
\begin{aligned}
E_{\perp}^{+++}= & -\left(J_{\perp}+\frac{1}{2} G_{\perp}\right) \cos \left(\phi_{1}-\phi_{2}\right) \\
& -\left(J_{\perp}^{\prime}+\frac{1}{2} G_{\perp}^{\prime}\right)\left[\cos \left(\phi_{1}-\phi_{3}\right)+\cos \left(\phi_{2}-\phi_{3}\right)\right],
\end{aligned}
$$




$$
\begin{aligned}
E_{\perp}^{++-}= & -\left(J_{\perp}+\frac{1}{2} G_{\perp}\right) \cos \left(\phi_{1}-\phi_{2}\right) \\
& +\frac{1}{2} G_{\perp}^{\prime}\left[\cos \left(\phi_{1}+\phi_{3}\right)+\cos \left(\phi_{2}+\phi_{3}\right)\right], \\
E_{\perp}^{-++}= & -\left(J_{\perp}^{\prime}+\frac{1}{2} G_{\perp}^{\prime}\right) \cos \left(\phi_{2}-\phi_{3}\right) \\
& +\frac{1}{2} G_{\perp} \cos \left(\phi_{1}+\phi_{2}\right)+\frac{1}{2} G_{\perp}^{\prime} \cos \left(\phi_{1}+\phi_{3}\right) .
\end{aligned}
$$

In the derivation of these results, use has been made of the relation

$$
\left(\frac{1}{N}\right) \sum_{n} e^{6 i \alpha n}=\delta(6 \alpha-2 \pi m),
$$

where $N$ is the periodicity along the columns. It is assumed here that $\alpha \neq m \pi / 3$, as appropriate for HHTT, so that the sum (14) is zero. Note from (12) and (13) that $E_{\perp}^{++-}$is independent of $J_{\perp}^{\prime}$ and that $E_{\perp}^{-++}$is independent of $J_{\perp}$. The relative stability of the three states was determined by numerically minimizing each energy expression (11)-(13) as a function of $\phi_{1}, \phi_{2}, \phi_{3}$ and comparing the results. The phase diagram corresponding to the ordinary triangular lattice, $J_{\perp}^{\prime}=J_{\perp}$ and $G_{\perp}^{\prime}=G_{\perp}$ so that states ++- and -++ are equivalent, is shown in Fig. 3. Four example $G_{\perp}-G_{\perp}^{\prime}$ phase diagrams using values $J_{1}= \pm 1.0$ and $J_{1}^{\prime}= \pm 0.6$ are shown in Fig. 4. The important observations to be made regarding these results are that each of the three possible chiral states can occur for certain ranges of parameter values and in addition, only the +++ state is stabilized if $G_{\perp}=G_{\perp}^{\prime}=0$.

As could be anticipated from the structure of the energies (11)-(13), large regions of the phase diagrams display states with $\phi_{p} \pm \phi_{p^{\prime}}=0$ or $\pi$; however, many other regions

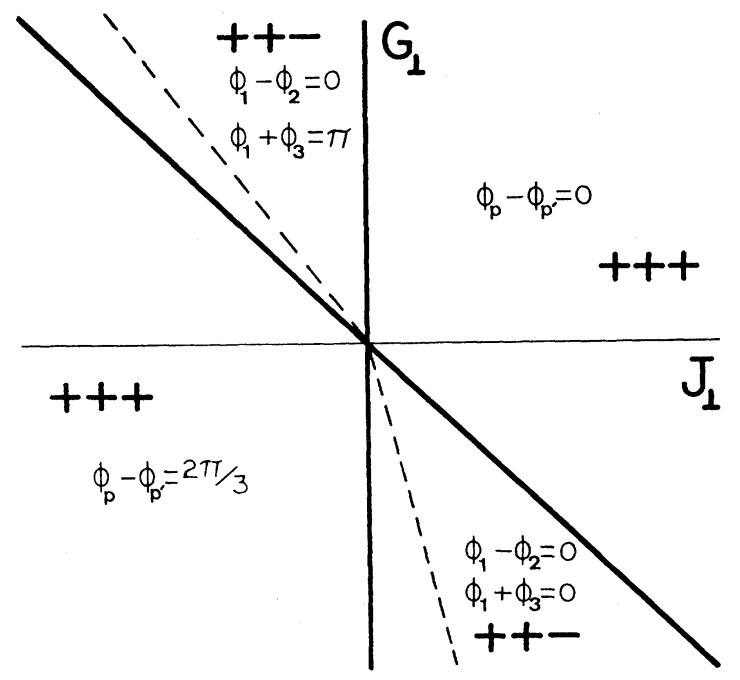

FIG. 3. Schematic ground-state phase diagram for the case $J_{\perp}^{\prime}=J_{\perp}, G_{\perp}^{\prime}=G_{\perp}$. Thick solid lines denote first-order transitions and broken lines represent continuous transitions. Chiralities of columns 1,2 , and 3 are indicated by + or - . Also shown are the relative phase angles of the first layer $\phi_{p}=3 \theta_{p}$. For the regions between the broken and solid lines of the ++- state, $\phi_{1}+\phi_{3}=-\left(\phi_{2}+\phi_{3}\right)$. Note that,++-+-+ , and -++ states are degenerate in this case. exhibit effects of frustration. Note also that the -++ state occurs only for cases (c) and (d) where $J_{1}<0$. Guided by these numerical results, it is straightforward to derive analytic expressions for most of the phase boundaries using (11)-(13). For example, the boundary between phases +++ and ++- , indicated by the solid horizontal line in each Fig. 4, is determined by $J_{\perp}^{\prime}+G_{\perp}^{\prime}=0$. The upper and lower broken phaseboundary lines in Fig. 4(a) are given by $G_{\perp}^{\prime}=-2 G_{\perp}-4 J_{\perp}-2 J_{\perp}^{\prime}$ and $G_{\perp}^{\prime}=2 G_{\perp}+4 J_{\perp}$, respectively. The intersection of the three chiral states in Figs. 4(c) and $4(\mathrm{~d})$ is at $G_{\perp}=-J_{\perp}, G_{\perp}^{\prime}=-J_{\perp}^{\prime}$. The symmetry between states with $J_{1}^{\prime}>0$ and $J_{1}^{\prime}<0$, evident by comparing the results of Figs. 4(a) with 4(b) as well as Figs. 4(c) and 4(d) can also be shown analytically from relations (11)-(13).

In the absence of some microscopic understanding of the model Hamiltonian, it is not possible to a priori assign realistic values for the parameters which might correspond with HHTT. However, the $H$ phase of this material appears to be in the ++- state with $\theta_{1}=\theta_{2}$ and $\theta_{3}$ taking a value $\pi / 3$ relative to the other two sites ${ }^{7}$ (see Fig. 1). This is precisely the state shown in the upper right-hand region of Fig. 4(b). It is thus clear that the $G$ terms in the Hamiltonian are crucial for a realistic model of the relative chirality and orientation of the helical columns in HHTT.

\section{MOLECULAR-FIELD THEORY}

Finite-temperature effects are examined here by analysis of a Landau-type free energy derived from the Hamiltonian (6) using the molecular-field approximation. For this purpose, it is convenient to express (6) in terms of pseudospin variables

$$
S_{x}\left(\mathbf{R}_{i}\right)=\cos 3 \theta_{i}, \quad S_{y}\left(\mathbf{R}_{i}\right)=\sin 3 \theta_{i},
$$

so that

$$
\begin{aligned}
H= & -\frac{1}{2} \sum_{i j} J\left(\mathbf{R}_{i}-\mathbf{R}_{j}\right) \mathbf{S}\left(\mathbf{R}_{i}\right) \cdot \mathbf{S}\left(\mathbf{R}_{j}\right) \\
& -\frac{1}{2} \sum_{i j} G\left(\mathbf{R}_{i}-\mathbf{R}_{j}\right) S_{y}\left(\mathbf{R}_{i}\right) S_{y}\left(\mathbf{R}_{j}\right) .
\end{aligned}
$$

The mean-field method of Bak and von Boehm ${ }^{20}$ may be generalized to the present system ${ }^{12,19}$ (or the densitymatrix formulation as described in Ref. 4) to yield a free energy expressed as an expansion in powers of the thermal average $\langle\mathbf{S}(\mathbf{R})\rangle=\mathbf{s}(\mathbf{R})$, which to sixth order is given by

$$
\begin{aligned}
F= & -\frac{1}{2} \sum_{i j} J\left(\mathbf{R}_{i}-\mathbf{R}_{j}\right) \mathbf{s}\left(\mathbf{R}_{i}\right) \cdot \mathbf{s}\left(\mathbf{R}_{j}\right) \\
& -\frac{1}{2} \sum_{i j} G\left(\mathbf{R}_{i}-\mathbf{R}_{j}\right) s_{y}\left(\mathbf{R}_{i}\right) s_{y}\left(\mathbf{R}_{j}\right) \\
& +T \sum_{i}\left\{\left[\mathbf{s}\left(\mathbf{R}_{i}\right)\right]^{2}+(1 / 4)\left[\mathbf{s}\left(\mathbf{R}_{i}\right)\right]^{4}\right. \\
& \left.+(5 / 36)\left[\mathbf{s}\left(\mathbf{R}_{i}\right)\right]^{6}+\cdots\right\} .
\end{aligned}
$$

Following our previous analyses of such free energies, an 
expansion of the spin density as a single Fourier component is assumed:

$$
\mathbf{s}_{p}(\mathbf{R})=\mathbf{S}_{p} e^{i \mathbf{Q}_{p} \cdot \mathbf{R}}+\mathbf{S}_{p}^{*} e^{-i \mathbf{Q}_{p} \cdot \mathbf{R}},
$$

where $\mathbf{R}=\mathbf{R}_{i}+\mathbf{u}_{p}, \mathbf{R}_{i}$ are the simple hexagonal lattice sites and $\mathbf{u}_{p}$ denotes the three basis vectors: $\mathbf{u}_{1}=a_{0} \hat{\mathbf{x}}, \mathbf{u}_{2}=2 a_{0} \hat{\mathbf{x}}, \mathbf{u}_{3}=\frac{1}{2} c \hat{\mathbf{z}}$. The wave vectors are assumed to have the simple form $\mathbf{Q}_{p}=c_{p}(3 \alpha / c) \hat{\mathbf{z}}$ and the complex polarization vectors can be written as

$$
\mathbf{S}_{p}=(1 / \sqrt{2}) S_{p}(\widehat{\mathbf{x}}-i \hat{\mathbf{y}}) e^{i \phi_{p}}
$$

Using these expressions, the free energy $F=F\left[S_{p}, \phi_{p}\right]$ with only close-neighbor interactions included (as described in the previous section) can be written as
$F=U_{\|}+U_{\perp}+T \sum_{p}\left[S_{p}^{2}+S_{p}^{4}+(20 / 3) S_{p}^{6}+\cdots\right]$,

where $U_{\|}=-J_{c} \Sigma_{p} S_{p}^{2}$, with $J_{c}=J_{\|}$for the chiral model (8) and $J_{c}=J_{1} \cos (Q c)+J_{2} \cos (2 Q c)$ for the nextnearest-neighbor model (9). $U_{\perp}$ depends on the relative chirality of the three chains as (11)-(13) with amplitude factor $S_{p}$ also included. For example, in the +++ state, the contribution to $U_{\perp}$ is given by

$$
\begin{aligned}
U_{\perp}^{+++}= & -\left(J_{\perp}+\frac{1}{2} G_{\perp}\right) S_{1} S_{2} \cos \left(\phi_{1}-\phi_{2}\right) \\
-\left(J_{\perp}^{\prime}+\frac{1}{2} G_{\perp}^{\prime}\right)[ & S_{1} S_{3} \cos \left(\phi_{1}-\phi_{3}\right) \\
& \left.+S_{2} S_{3} \cos \left(\phi_{2}-\phi_{3}\right)\right],
\end{aligned}
$$
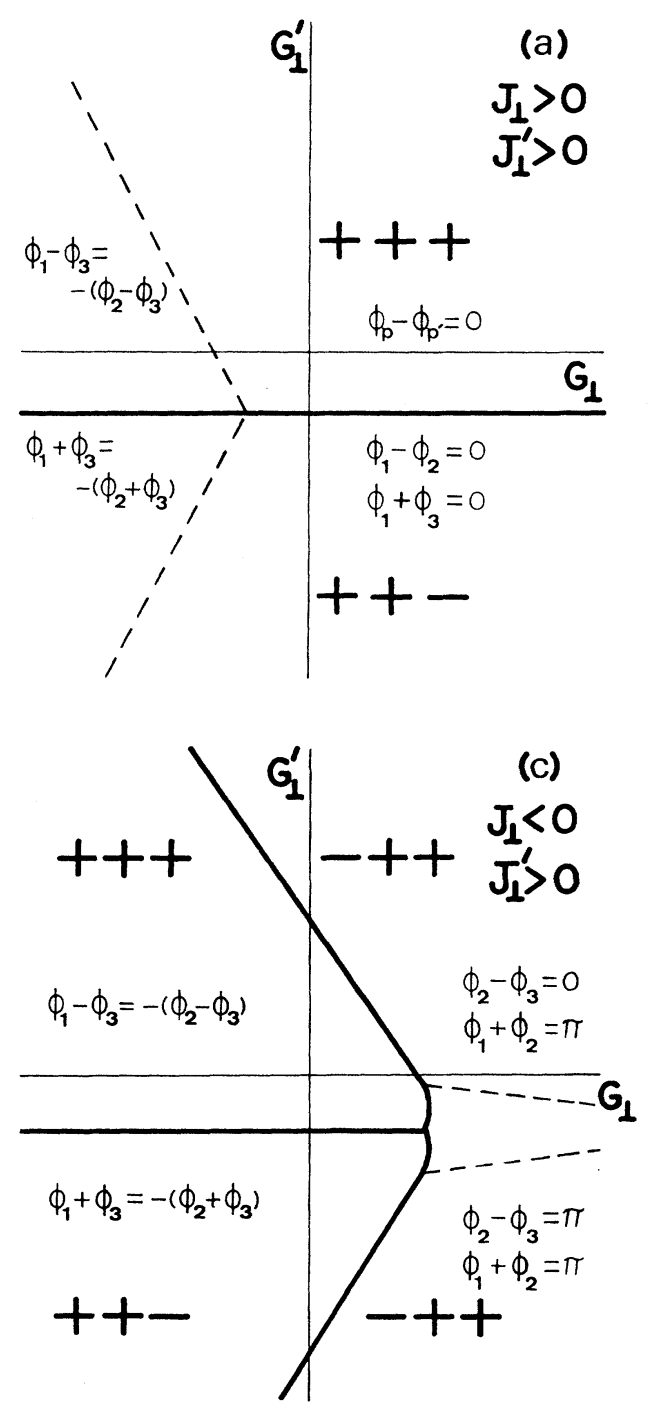
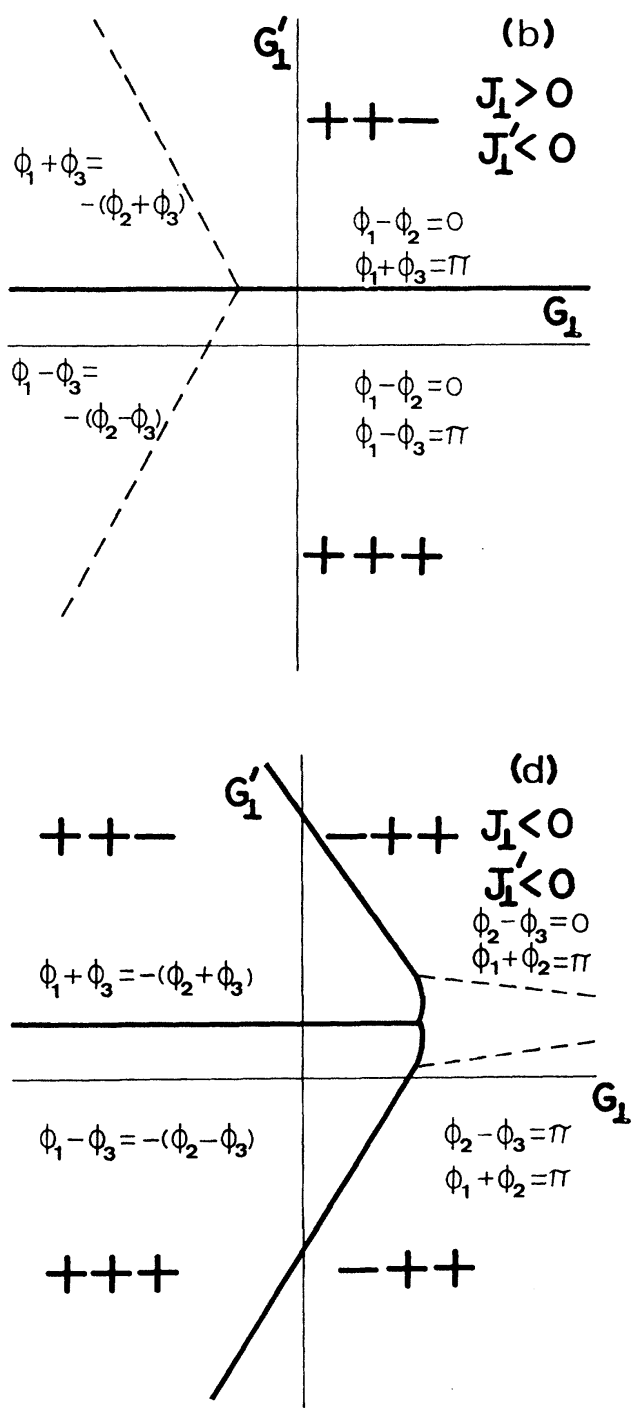

FIG. 4. Representative ground-state phase schematics constructed using $J_{\perp}= \pm 1.0$ and $J_{\perp}^{\prime}= \pm 0.6$, labelled as in Fig. 2 . For the regions between the broken lines of the -++ state in Figs. 3(c) and 3(d), there is no simple relationship among the $\phi_{p}$. 
with analogous results for the other two chiral states. It is important to note, as with all such finite Landau expansions, that the results described below are reliable only at temperatures not too far from the disordered state.

Within this model, there is a continuous transition between the disordered $D_{h d}$ phase, characterized here by $S_{1}=S_{2}=S_{3}=0$, and the $H$ phase where one or more of the $S_{p}$ are nonzero. Illustrative examples of $G_{\perp}-T$ phase diagrams for the case $J_{1}^{\prime}=J_{1}, G_{1}^{\prime}=G_{1}$ are presented in Fig. 5. The results at low temperature can be seen to correspond roughly with the ground-state phase diagram, Fig. 3. In this case, all three of the columns are always ordered $\left(S_{p}>0\right)$. Three example $G_{\perp}^{\prime}-T$ phase diagrams are shown in Figs. 6-8 for various values of $J_{\perp}, J_{\perp}^{\prime}$, and $G_{\perp}$. The low-temperature results of Fig. 6 correspond to a vertical cut in the ground-state phase diagram Fig. 4(b); however, a new feature appears at higher temperatures. In the region between the,+++++- , and disordered states, a partially disordered $(++)$ phase appears, characterized by long-range orientational order on columns 1 and 2 but not on column $3\left(S_{3}=0\right)$. (A similar magnetic state appears in some $A B X_{3}$ compounds. ${ }^{12}$ ) It was originally believed ${ }^{6}$ that interdigital frustration was relieved in HHTT by the $H$ phase characterized in this way, but further data analysis ${ }^{7}$ now favors the ++-
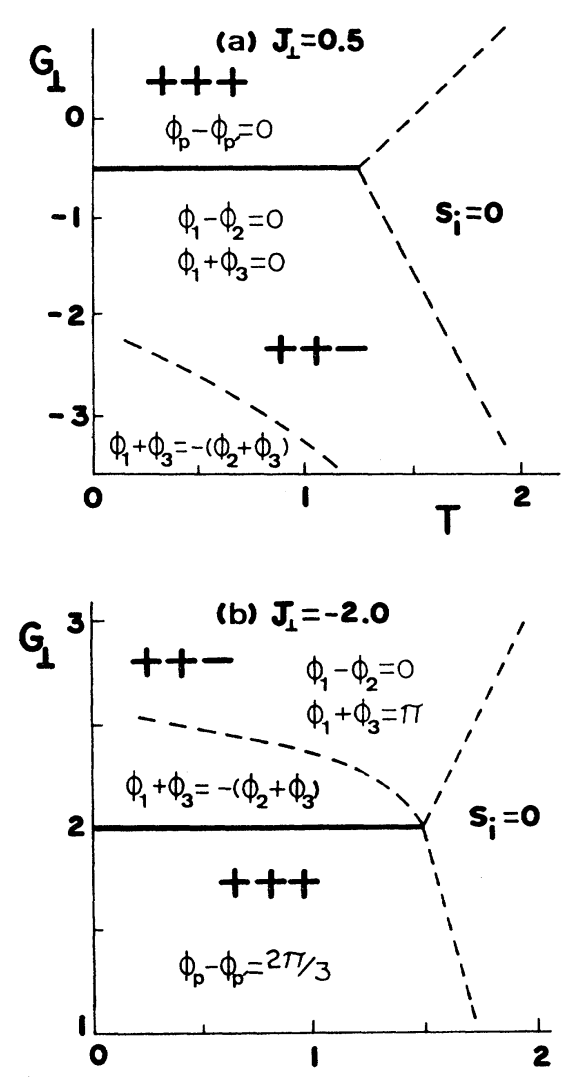

FIG. 5. Examples from the temperature-dependent phase diagram for the case $J_{\perp}^{\prime}=J_{\perp}, G_{\perp}^{\prime}=G_{\perp}$ from molecular-field theory, labeled as in Fig. 2. The high-temperature state with no orientational order is indicated by $S_{p}=0$.

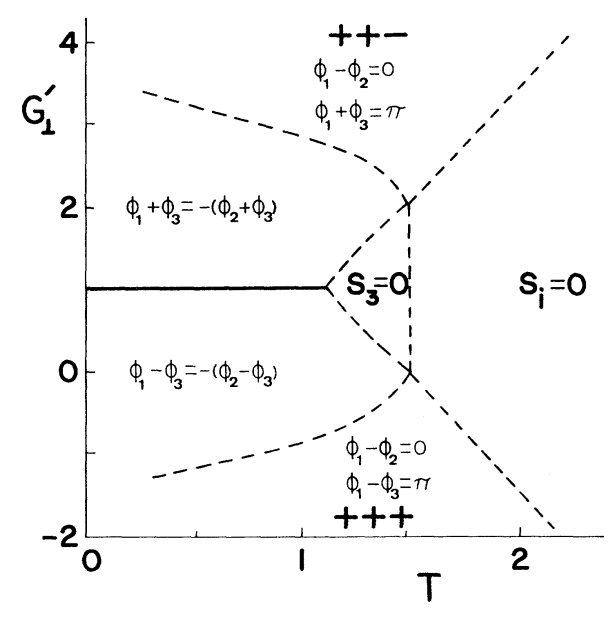

FIG. 6. Phase diagram for the case $J_{\perp}=+1, J_{\perp}^{\prime}=-1$, $G_{1}=-4$. The partially disordered phase indicated by $S_{3}=0$ is in a ++ relative chirality state, where $\phi_{1}-\phi_{2}=\pi$.

state. Within the present model, as seen from Fig. 6, only a small change in the parameter $G_{\perp}^{\prime}$ is required to stabilize either of these two states.

The $G_{\perp}^{\prime}-T$ phase diagrams of Fig. 7 and 8 correspond to parameter values of the ground-state phase diagram Fig. 4(c). The value of $G_{\perp}$ used to construct Fig. 7 is large enough so that only the -++ state occurs, except along the horizontal line where a partially disordered phase -+ is stabilized. A $S_{3}=0$ state $(++)$ is also seen to occur for the parameter values of Fig. 8. These results demonstrate that a variety of phase-transition sequences are possible with varying temperature. Unlike magnetic systems, it can be expected here that the couplings between columns have relatively strong thermal dependence due to the stiffening of the hydrocarbon tails as the temperature is lowered, ${ }^{14}$ a feature which gives rise to anomalous behavior in the thermal expansion. ${ }^{6-8}$ Thus, the

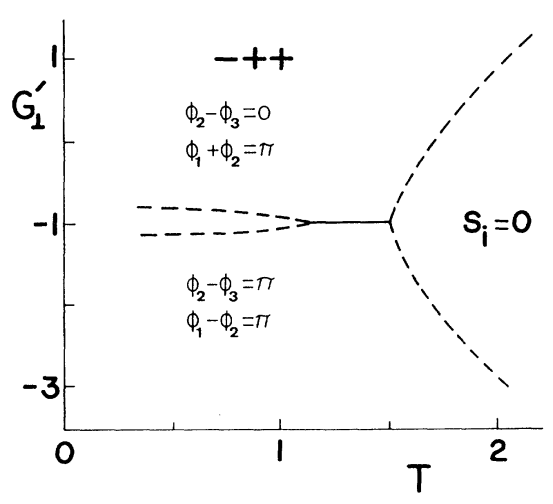

FIG. 7. Phase diagram for the case $J_{1}=-1, J_{\perp}^{\prime}=+1$, $G_{1}=+2$. In the region between the broken lines of the -++ state, there is no simple relationship among the $\phi_{p}$. A partially disordered -+ phase, $S_{3}=0$, occurs along the sold line. 


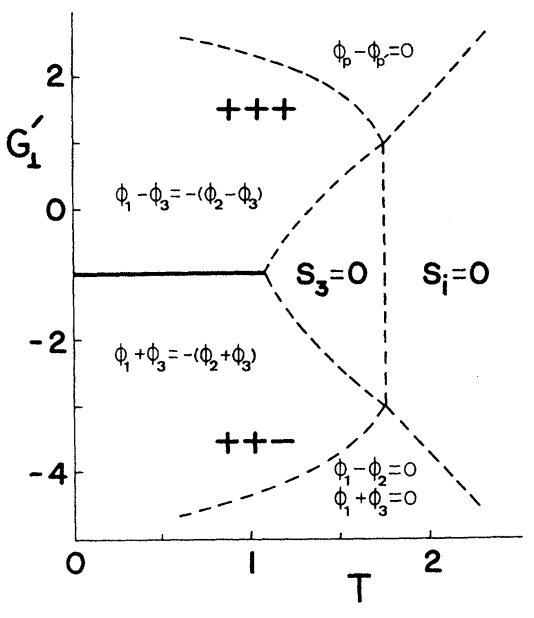

FIG. 8. Phase diagram for the case $J_{1}=-1, J_{1}^{\prime}=+1$, $G_{\perp}=-1$. The partially disordered ++ phase is indicated by $S_{3}=0$, where $\phi_{1}-\phi_{2}=\pi$.

effect of varying temperature on these model phase diagrams may not necessarily be taken as simple horizontal straight-line cuts.

\section{CONCLUSIONS}

It has been demonstrated by this work that an interaction ( $G$ term) unique to octupolar disks coupled in a triangular array is responsible for a variety of possible relative-chirality states associated with helically oriented columns. For the case of interacting honeycomb and triangular lattices relevant for HHTT, all five of the possible states considered in Ref. $7,+++,++-,-++$, and the two finite-temperature partially disordered states
++ and -+ , are a feature of this model. This result contrasts with the realization of only the +++ state in the absence of the $G$ term. An indication of the range of parameter values required to stabilize the observed ++- state can be made from easily obtained analytic expressions for the phase boundaries associated with the ground-state phase diagrams of Fig. 4. The possibility that there may be phase transitions within the helical phase of HHTT between other relative-chirality or relative phase-angle $\left(\phi_{p}\right)$ states should be considered in view of the representative temperature phase diagrams of Figs. 6-8. Determination of the nature of the helical phase in HHTT compounds with differing numbers of hydrocarbon-chain tails and with varying samplepreparation procedures ${ }^{9}$ is also of interest.

Frustration of interdigitated hydrocarbon tails is believed ${ }^{6-8}$ responsible for the displacement by $\frac{1}{2} c$ of onethird of the columns at precisely the temperature of the $H-D_{h d}$ transition. Although omitted from the present study, this aspect associated with the onset of long-range orientational order in HHTT is clearly of interest. It is for this reason that the transition is first order in character, and the manifestations of the possibly novel critical behavior would be difficult to observe in this material. A model which allows for displacements of the columns and includes coupling between orientational order and this lattice degree of freedom, and which demonstrates that this structural/orientational phase transition is driven by the triangular frustration, is desirable.

\section{ACKNOWLEDGMENTS}

We thank A. B. Harris, M. Hébert, P. Heiney, S. Idziak, and J. Rimers for useful discussions and correspondence. Work was supported by the Natural Sciences and Engineering Research Council (NSERC) of Canada and le Fonds FCAR pour l'Aide et le Soutien á la Recherche du Québec.
${ }^{1}$ R. S. Gekht and V. I. Ponomarev, Phys. Trans. 20, 27 (1990); K. Hirakawa, ibid. 28, 33 (1990).

${ }^{2}$ M. L. Pumer and A. Caillé, J. Appl. Phys. 70, 5961 (1991).

${ }^{3}$ H. Kawamura, J. Phys. Soc. Jpn. 61, 1299 (1992).

${ }^{4}$ H.-Y. Choi, A. B. Harris, and E. J. Mele, Phys. Rev. B 40, 3766 (1989).

${ }^{5}$ O. Heinonen and P. L. Taylor, Polymer 32, 2155 (1991); O. Heinonen, Phys. Rev. B 47, 2661 (1993).

${ }^{6}$ E. Fontes, P. A. Heiney, and W. H. de Jeu, Phys. Rev. Lett. 61, 1202 (1988).

${ }^{7}$ P. A. Heiney, E. Fontes, W. H. de Jeu, A. Riera, P. Carroll, and A. B. Smith, J. Phys. (Paris) 50, 461 (1989).

${ }^{8}$ E. Fontes, Ph.D. thesis, University of Pennsylvania, 1989.

${ }^{9}$ S. H. Idziak, Ph.D. thesis, University of Pennyslvania, 1992; S. H. Idziak, P. A. Heiney, J. P. McCauley, Jr., P. Carroll, and A. B. Smith III, Mol. Cryst. Liq. Cryst. Lett. (to be pub- lished).

${ }^{10}$ S. Chandrasekhar and G. S. Ranganath, Rep. Prog. Phys. 53, 57 (1990).

${ }^{11}$ M. Hébert, M. S. thesis, Université de Sherbooke, 1992.

${ }^{12}$ M. L. Plumer, A. Caillé, and H. Kawamura, Phys. Rev. B 44, 4461 (1991).

${ }^{13} \mathrm{M}$. Hébert and A. Caillé (unpublished).

${ }^{14}$ P. A. Heiney (private communication).

${ }^{15}$ O. Heinonen and P. L. Taylor, Polymer 30, 585 (1989).

${ }^{16}$ S. Hirotsu, J. Phys. C 10, 967 (1977); H. Tanaka, U. Schotte, and K. D. Schotte, J. Phys. Soc. Jpn. 61, 1344 (1992).

${ }^{17}$ M. Pesquer, M. Cotrait, P. Marsau, and V. Volpilhac, J. Phys. (Paris) 41, 1039 (1980).

${ }^{18}$ Y. Saruyama, J. Chem. Phys. 78, 2077 (1983).

${ }^{19}$ M. L. Plumer, Phys. Rev. B 44, 12376 (1991).

${ }^{20}$ P. Bak and J. von Boehm, Phys. Rev. B 21, 5297 (1980). 